\title{
Intravascular Lymphoma in the CNS: Options for Treatment
}

\author{
Damir Nizamutdinov, MD, $P h D^{1,2}$ \\ Nitesh P. Patel, $B S^{1}$ \\ Jason H. Huang, $M D^{1,2}$ \\ Ekokobe Fonkem, DO $0^{1,2, *}$
}

\author{
Address \\ ${ }^{1}$ College of Medicine, Texas A\&M Health Science Center, Temple, TX, USA \\ ${ }^{*}, 2$ Department of Neurosurgery, Baylor Scott \& White Health, Temple, TX, USA \\ Email: Ekokobe.Fonkem@BSWHealth.org
}

Published online: 23 August 2017

(C) The Author(s) 2017. This article is an open access publication

This article is part of the Topical Collection on Cerebrovascular Disorders

Damir Nizamutdinov and Nitesh P. Patel equally contributed as first authors.

Keywords Intravascular lymphoma $\cdot$ CNS · Treatment

\begin{abstract}
Purpose of review The purpose of this review was to discuss therapeutic manipulations and effective current interventions available to treat intravascular lymphoma in the central nervous system.

Recent findings Patients experienced resolution and remission of disease for 14 months and up to 2 years after eight cycles of R-CHOP and four courses of intrathecal therapy with MTX, cytarabine, and prednisolone. Intravascular use of unfractionated heparin during therapy may contribute to better outcome.

Summary Series of therapeutic avenues were analyzed and compared. The effective current treatment of intravascular lymphoma in the CNS is considered to be a combinational intrathecal methotrexate-based chemotherapy with rituximab. Since intrathecal administration bypasses the blood-brain barrier, lower doses can be given, which thereby minimizes systemic toxicity. Practical use of intrathecal chemotherapy is also justified for prophylaxis in intravascular lymphoma-diagnosed patients without CNS involvement.
\end{abstract}

\section{Introduction}

Intravascular lymphoma (IVL) is a rare form of extranodal non-Hodgkin's lymphoma that selectively involves the lumina of small blood vessels, especially capillaries [1]. The disorder was initially characterized in
1959 as a neoplasm of vascular endothelial cells and was thereby named "angioendotheliomatosis proliferans systemisata" [2]. However, the lymphoid origin was elucidated by immunohistochemistry in 1986; at that 
time, the disorder was renamed "angiotropic large cell lymphoma," and the nomenclature was further refined to "intravascular lymphoma" by the World Health Organization in 2008 [3, 4]. While the majority of IVL is of the B lymphocyte lineage, rare cases have been observed involving the natural killer (NK)/T cell lineage [5].

Given the intravascular (IV) proliferation of neoplastic lymphocytes, the disorder typically presents with signs and symptoms of vascular occlusion and thus can have a variable clinical presentation depending on

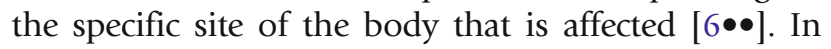
2014, Fonkem et al. performed a meta-analysis of 740 IVL cases and showed that the central nervous system (CNS) is the most common location (41\%) of primary disease $[7 \bullet \bullet]$. However, previous reports suggested that clinical manifestations also vary based on a patient's geographic origin. Specifically, involvement of the CNS and skin is observed at a greater frequency in Western countries, whereas patients in Asian countries typically present with fever, hematophagocytic syndrome, hepatosplenomegaly, and bone marrow invasion [8].

All cases of IVL are managed as disseminated disease due to the IV nature of the disorder, and the most common treatment regimen consists of cyclophosphamide, doxorubicin, vincristine, prednisone, and rituximab (R-CHOP) [1]. Clinical evidence for this combination is mainly derived from case reports and retrospective studies, and long-term survival evidence has been limited due to the short follow-up in many of these studies [1, 9-13]. Furthermore, given that systemic treatment with R-CHOP does not adequately penetrate the CNS, further CNS-directed intervention is warranted given that approximately $30-40 \%$ of patients have CNS involvement at the time of diagnosis and another $25 \%$ develop CNS involvement during the follow-up period [14]. This review mainly focuses on therapeutic options and managements of IVL in the CNS.

\section{Methods}

Literature search: We conducted a literature search of PubMed from 1962 to March 2017 using the following terms: intravascular with lymphoma or lymphomatosis, angioendotheliomatosis, endotheliomatosis, and large cell lymphoma with angiotropic or angiotrophic. Case reports of IVL with CNS involvement were included, but reports that did not comment on therapy of CNS involvement or patient outcomes were excluded. Publications in a nonEnglish language were also excluded from this review.

\section{Clinical manifestations and diagnosis}

\section{Clinical manifestations}

Clinical presentation of IVL with CNS involvement depends on location and severity of cerebrovascular pathology associated with disease. Aforementioned pathology compromises optimal blood supply to important brain structures and results in impaired brain function. IVL limited only to the CNS is an extremely rare condition. In 2016, Fonkem et al. performed a meta-analysis of 654 IVL cases with neurological presentations and reported that $52 \%$ of all IVL-diagnosed patients were presented with neurologic symptoms and neurologic complications [15]. Of them, $82 \%$ were presented with CNS symptoms, $18 \%$ with peripheral nervous system (PNS) deficits. Most commonly observed CNS complication was cognitive impairment (60.9\%), including encephalopathy and dementia. Paralysis (upper motor neuron) and paraplegia were second most commonly reported CNS symptoms (22.1\%). Seizures, including myoclonic jerks and status epilepticus, were third most common clinical symptoms (13.4\%) of CNS involvement [15]. Among other reported symptoms were 
vision disturbances $(8.7 \%)$, ataxias (7.6\%), stroke-like symptoms (7.6\%), headaches (6.9\%), myelopathies (5.8\%), dysarthrias (5.4\%), sensory and hearing deficits (4.7 and $4.4 \%$, respectively), and others [15].

\section{Diagnosis}

Unfortunately, due to lack of diagnostic algorithms and very diverse clinical presentation, $60 \%$ of IVL patients with CNS involvement are diagnosed postmortem $[15,16]$. IVL is rare disease, but when a patient demonstrates rapid development of progressive neurological manifestations without logical correlation to previous history of disease, IVL should be suspected until more clinical diagnostic tests are performed.

Radio-diagnostic criteria found useful in diagnostics of IVL with CNS involvement. Neuro-radiological images are characterized by absence of uniform radiological findings. Thus, MRI should be routinely made to all suspects to rule out more common neurologic disease. Neurosurgical biopsy performed in timely manner is highly recommended and serves as an accurate diagnostic tool, but should be used sparingly in the brain, due to invasiveness $[15,17]$. Since IVL is systemic pathology, biopsy from other easily accessible sites should be considered [17]. Laboratory findings reveal a pathologic hemogram in most patients. Usually, thrombocytopenia $(29 \%)$ or leukopenia $(24 \%)$ does not occur without developed anemia (63\%) [17]. Increased serum lactic dehydrogenase and beta-2 microglobulin levels were observed in more than $80 \%$ of patients [9]. Combination of diagnostic approaches is needed to timely confirm a diagnosis and start adequate therapeutic regimen.

\section{Therapeutic interventions}

Due to the rarity of IVL, no prospective, randomized trials have been performed to evaluate the optimal treatment of CNS involvement; instead, support for current methods is derived from case reports, retrospective studies, and trials of more common lymphomas affecting the CNS [18].

Intrathecal chemotherapy involves injection of chemotherapeutic agents directly to cerebrospinal fluid within the intrathecal space, thereby eliminating the need for the drugs to cross the blood-brain barrier (BBB). This method has been employed in recent cases of IVL as both CNS treatment and prophylaxis $[19-21 \bullet, 26]$. The combination of methotrexate (MTX), cytarabine, and prednisolone given intrathecally has been shown to be effective in various degrees of CNS involvement [20, 21 •, 23]. In 2014, a Japanese patient with pontine involvement was the first reported case of IVL involving the CNS that achieved remission with only R-CHOP and intrathecal therapy. The patient experienced resolution of the pontine lesion and achieved remission for 14 months after eight cycles of R-CHOP and four courses of intrathecal therapy with MTX, cytarabine, and prednisolone [20]. More recently in 2016, a patient that developed IVL with involvement of the dura on brain magnetic resonance imaging (MRI) achieved complete remission for 2 years without CNS relapse after a similar treatment regimen [23]. Furthermore, a patient with IVL involving the cauda equina causing lower extremity paraplegia achieved complete 
remission for 28 months with the same R-CHOP and intrathecal chemotherapy combination, but her paraplegia persisted [21]. Intrathecal administration appears to be a good option for cauda equina involvement given that two other patients presenting with cauda equina syndrome both achieved clinical improvement and complete remission following intrathecal chemotherapy and systemic treatment with either R-CHOP or RCPADEM (rituximab, MTX, doxorubicin, endoxan, lederfolin, and prednisone) $[19,22]$.

Prophylaxis with intrathecal chemotherapy has resulted in varied outcomes [24-27]. Two cases of IVL without CNS involvement at diagnosis achieved complete remission without CNS relapse for 20 months in the patient that received four doses of intrathecal MTX and 21 months in the patient that received intrathecal MTX, cytarabine, and dexamethasone [25, 26]. However, even though a patient with IVL of the penis, prostate, and bones without CNS involvement at diagnosis achieved complete remission after six cycles of systemic treatment with R-CHOP and four doses of intrathecal MTX for CNS prophylaxis, the patient ultimately experienced CNS relapse after 6 months [24]. Overall, intrathecal chemotherapy has been effective as both treatment and prophylaxis of CNS involvement of IVL. Benefits of the intrathecal method include smaller drug doses due to the small volume of distribution of the CSF, which thereby minimizes systemic toxicity. Given that this treatment combination is better tolerated than other regimens, it may be an appropriate choice for treatment of elderly patients or patients in poor medical condition that cannot tolerate regimens with greater toxicity.

Despite the benefits of intrathecal chemotherapy, some patients with CNS involvement have responded to R-CHOP treatment alone [27-29]. A patient with IVL of pituitary gland who presented with features of pituitary apoplexy and ophthalmic symptoms achieved clinical and radiographic improvement with three cycles of R-CHOP. Although the patient continued to have right cranial nerve III palsy, the sellar mass resolved after R-CHOP therapy, indicating that IVL of the pituitary can be treated with R-CHOP alone [27]. In 2014, a Japanese patient without neurological symptoms was diagnosed with IVL of the pons after at T2 weighted brain MRI showed a pontine hyper-intensity. After eight cycles of R-CHOP without intrathecal therapy, the patient experienced resolution of the pontine lesion without recurrence for 5 years [28•]. This case indicates that R-CHOP is effective at treating IVL with early CNS involvement. Furthermore, it should be noted the patient also had taken verapamil for paroxysmal supraventricular tachycardia. Verapamil inhibits p-glycoprotein, which is an important mediator of resistance to anticancer drugs and also limits drug penetration through the BBB [30, 31]. Thus, early initiation of chemotherapy in addition to a pglycoprotein inhibitor like verapamil may be important in treating IV large B cell lymphoma with CNS involvement and is an area of possible study [28•]. However, another patient with pontine involvement of IVL experienced diminution of her pontine lesion after three cycles of R-CHOP alone and this patient was not taking verapamil concurrently [29]. While the utility of verapamil is questionable, these cases suggest that IVL of the pituitary and pons responds well to R-CHOP alone. Despite this, a case of IVL involving 
both the brain and spinal cord initially responded to five cycles of R-CHOP, but after 5 months, the patient experienced CNS recurrence with neurological symptoms, suggesting that R-CHOP alone may not be sufficient treatment for IVL with extensive CNS involvement [32].

While R-CHOP seems beneficial in cases of less extensive CNS involvement, especially of the pituitary gland and pons, CHOP therapy alone without rituximab has mostly led to poor outcomes [33, 34]. Two patients presenting with memory loss and behavioral changes with involvement of the brain on MRI both expired after treatment solely with CHOP, suggesting the need for CNSdirected therapy $[33,34]$. One of the patients initially received high-dose IV methylprednisolone for 5 days, which temporarily improved her neurological condition from a Glasgow coma scale (GCS) score of 7 to GCS of 11 [34]. Accordingly, most other cases utilizing high-dose corticosteroids have only observed a transient improvement in neurological condition and thus should not be the sole component of CNS treatment $[18,35,36]$.

In 2012, a case of IVL, affecting the pons and left temporomesial area in a Caucasian male, was treated with the Bonn protocol, which consists of six cycles of high-dose MTX, ifosfamide, procarbazin, cytarabine, vinca alkaloids, and dexamethasone in addition to rituximab $[37,38]$. The patient achieved complete clinical and radiographic remission for 29 months since diagnosis after receiving treatment, and although the protocol was developed for primary CNS lymphoma (PCNSL), the favorable outcome suggests that a high-dose MTX-based polychemotherapy is appropriate for treatment of IVL in the CNS [37]. Another regimen that has shown promise was recently reported by Prat-Martin et al. and consists of carmustine, cytarabine, and MTX. In this report, a case of IVL affecting the brain achieved complete remission for 17 months since diagnosis after two cycles of carmustine, cytarabine, and MTX, followed by one cycle of R-CHOP and stem cell support [39]. The favorable CNS response may be as result of the high penetration ability of BBB by carmustine, cytarabine, and MTX.

PCNSL is often treated with high-dose MTX followed by whole-brain radiotherapy, and although a few case reports have suggested treating the CNS involvement of IVL in a similar manner, the outcomes have been conflicting [35, 40]. In 2012, a patient with IVL limited to the CNS experienced radiographic and clinical improvement after three cycles of high-dose MTX followed by whole-brain radiotherapy at a total dose of $30 \mathrm{~Gy}$; however, the patient expired 6 months after disease onset [40]. Conversely, a patient with IVL of the dura on brain MRI was successfully treated with involved field radiotherapy after resistance to high-dose MTX [35]. While radiotherapy alone is not commonly employed in the therapy of CNS IVL, a patient with recurrent stroke-like symptoms experienced stabilization of intracranial lesions for 7 months after therapy with RCHOP, MTX, and cranial radiotherapy [41]. However, a patient that received a total of 45 Gy via whole-brain radiotherapy followed by R-COP (rituximab, cyclophosphamide, vincristine, and prednisone) experienced neurological deterioration 10 months after onset despite clinical and radiological improvement for 8 months since onset. The lack of CNSdirected chemotherapy in combination with radiotherapy in this patient 
could explain the clinical course, suggesting that intrathecal chemotherapy has greater utility than radiotherapy [42].

\section{Conclusion}

Recent case reports have presented novel treatment options for the CNS component of IVL $[24,43]$. In the aforementioned case of failed CNS prophylaxis with intrathecal MTX in the patient with IVL of the penis, prostate, and bones, CNS relapse significantly improved after three cycles of oral temozolomide with IV rituximab and high-dose MTX [24]. Also, in 2015, Yoshida et al. used IV unfractionated heparin and observed rapid improvement of the patient's white matter and auditory nerve lesions with improved hearing after 10 days of heparin initiation; furthermore, the patient achieved complete remission after five cycles of R-CHOP and

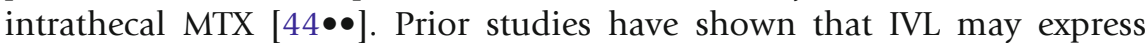
heparin-responsible adhesion molecules such as L-selectin and very late antigen-4; thus, treating with heparin could prevent the aggregation of the neoplastic lymphocytes within vascular lumina and thereby contribute to the treatment of CNS involvement without further contributing to the systemic toxicity of chemotherapeutic agents $[44 \bullet \bullet, 45]$.

At the present time, the most effective treatment of IVL in the CNS is a combination of MTX-based intrathecal chemotherapy and R-CHOP [19$26]$. The lower dose of administered drug can be used due to BBB penetration, and resulted in minimized systemic toxicity. Intrathecal chemotherapy should also be utilized as prophylaxis in patients without CNS involvement.

\section{Compliance with Ethical Standards}

Conflict of Interest

The authors declare that they have no conflict of interest.

Human and Animal Rights

This article does not contain any studies with human or animal subjects performed by any of the authors.

\section{Open Access}

This article is distributed under the terms of the Creative Commons Attribution 4.0 International License (http://creativecommons.org/licenses/by/4.0/), which permits unrestricted use, distribution, and reproduction in any medium, provided you give appropriate credit to the original author(s) and the source, provide a link to the Creative Commons license, and indicate if changes were made. 


\section{References and Recommended Reading}

Papers of particular interest, published recently, have been highlighted as:

- Of importance

- Of major importance

1. Ponzoni M, Ferreri AJM, Campo E, et al. Definition, diagnosis, and management of intravascular large Bcell lymphoma: proposals and perspectives from an international consensus meeting. J Clin Oncol. 2007;25:3168-73.

2. Pfleger V, Tappeiner J. On the recognition of systematized endotheliomatosis of the cutaneous blood vessels reticuloendotheliosis. Hautarzt. 1959;10:359-63.

3. Wick MR, Mills SE, Scheithauer BW, Cooper PH, Davitz MA, Parkinson K. Reassessment of malignant angioendotheliomatosis. Evidence in favor of its reclassification as intravascular lymphomatosis. Am J Surg Pathol. 1986;10:112-23.

4. Nakamura S, Ponzoni M, Campo E (2008) Intravascular large B-cell lymphoma. In: WHO Classif. Tumours Haematop. Lymphoid Tissues. pp 252-253.

5. Xie J, Zhou X, Zhang X, Zheng Y, Yue B. Primary intravascular natural killer/T cell lymphoma of the central nervous system. Leuk Lymphoma.

2014;8194:1-3.

6.• Rajyaguru DJ, Bhaskar C, Borgert AJ, Smith A, Parsons B. Intravascular large B-cell lymphoma in the United States (US): a population-based study using Surveillance, Epidemiology, and End Results program and National Cancer Database. Leuk Lymphoma. 2017;0:1-9.

This review is based on the US National Cancer Database and conducted to determine incidence, demographic and clinical characteristics, prognostic factors, and survival for intravascular large B cell lymphoma.

7.• Fonkem E, Lok E, Robison D, Gautam S, Wong ET. The natural history of intravascular lymphomatosis. Cancer Med. 2014;3:1010-24.

This meta-analysis covers large group of people and discusses treatment regimens along with survival outcomes for treatment of IVL inside and outside of CNS.

8. Murase T, Nakamura S, Kawauchi K, Matsuzaki H, Sakai C, Inaba T, et al. An Asian variant of intravascular large B-cell lymphoma: clinical, pathological and cytogenetic approaches to diffuse large B-cell lymphoma associated with haemophagocytic syndrome. $\mathrm{Br} \mathrm{J}$ Haematol. 2000;111:826-34.

9. Ferreri AJM, Campo E, Seymour JF, et al. Intravascular lymphoma: clinical presentation, natural history, management and prognostic factors in a series of 38 cases, with special emphasis on the cutaneous variant. Br J Haematol. 2004;127(2):173-83.

10. Shimada K, Matsue K, Yamamoto K, et al. Retrospective analysis of intravascular large B-cell lymphoma treated with rituximab-containing chemotherapy as reported by the IVL Study Group in Japan. J Clin Oncol. 2008;26:3189-95.

11. Ferreri AJM, Dognini GP, Govi S, et al. Can rituximab change the usually dismal prognosis of patients with intravascular large B-cell lymphoma? J Clin Oncol. 2008;26:3189-95.

12. Bouzani M, Karmiris T, Rontogianni D, Delimpassi S, Apostolidis J, Mpakiri M, et al. Disseminated intravascular B-cell lymphoma: clinicopathological features and outcome of three cases treated with anthracyclinebased immunochemotherapy. Oncologist. 2006;11:923-8.

13. Koizumi M, Nishimura M, Yokota A, Munekata S, Kobayashi T, Saito Y. Successful treatment of intravascular malignant lymphomatosis with high-dose chemotherapy and autologous peripheral blood stem cell transplantation. Bone Marrow Transpl. 2001;27:1101-3.

14. Shimada K, Murase T, Matsue K, et al. Central nervous system involvement in intravascular large B-cell lymphoma: a retrospective analysis of 109 patients. Cancer Sci. 2010;101:1480-6.

15. Fonkem E, Dayawansa S, Stroberg E, et al. Neurological presentations of intravascular lymphoma (IVL): metaanalysis of 654 patients. BMC Neurol. 2016;16:9.

16. Hundsberger T, Coqliatti S, Kleger GR, et al. Intravascular lymphoma mimicking cerebral stroke: report of two cases. Case Rep Neurol. 2011;3(3):278-83.

17. Ponzoni M, Ferreri AJ. Intravascular lymphoma: a neoplasm of 'homeless' lymphocytes? Hem Oncol. 2006;24:105-12.

18. Zuckerman D, Seliem R, Hochberg E. Intravascular lymphoma: the oncologist's "great imitator". Oncologist. 2006;11:496-502.

19. Coulibaly B, Mesturoux L, Petit B, Magy L, Labrousse F. Intravascular large B-cell lymphoma presenting as cauda equina syndrome and showing aberrant cytokeratin expression: a diagnostic challenge. Pathology. 2014;46:241-4.

20. Sekiguchi $Y$, Shimada A, Imai H, Wakabayashi M, Sugimoto K, Nakamura N, et al. Intravascular large Bcell lymphoma with pontine involvement successfully treated with R-CHOP therapy and intrathecal administration: a case report and review of literature. Int J Clin Exp Pathol. 2014;7:3363-9.

21. Tsuda M, Nakashima Y, Ikeda M, et al. Intravascular large B-cell lymphoma complicated by anti-neutrophil cytoplasmic antibody-associated vasculitis that was successfully treated with rituximab-containing chemotherapy. J Clin Exp Hematop. 2015;55:39-43. 
This report discusses successful treatment of complicated IVL BCL case.

22. Colchester NTH, Barker CS, Jogai S, Katifi H a. Cauda equina syndrome due to intravascular lymphoma: diagnosis by nasal biopsy. Pract Neurol. 2015;15:210-3.

23. Kikuchi J, Kaneko Y, Kasahara H, Emoto K, Kubo A, Okamoto S, et al. Methotrexate-associated intravascular large B-cell lymphoma in a patient with rheumatoid arthritis. Intern Med. 2016;55:1661-5.

24. Wakim JJ, Levenson BM, Mathews D, Naina HV. Management of an unusual case of intravascular large B-cell lymphoma of the penis, prostate, and bones with CNS relapse. J Clin Oncol. 2013;31:e288-90.

25. Ramkumar B, Hutchison R, Khadim H, Gajra A. Intravascular large B-cell lymphoma-a diagnostic dilemma. Clin Lymphoma Myeloma Leuk. 2013;13:e18-21.

26. Yamamoto K, Yakushijin K, Okamura A, Hayashi Y, Matsuoka H, Minami H. Gain of 11q by an additional ring chromosome 11 and trisomy 18 in CD5-positive intravascular large B-cell lymphoma. J Clin Exp Hematop. 2013;53:161-5.

27. Rizek P, Seitelbach M, Alturkustani M, Leung A, Fraser JA. Sellar and parasellar intravascular lymphoma mimicking pituitary apoplexy. J Neuro-Ophthalmology. 2012;32:33-7.

28. Sawada T, Omuro Y, Kobayashi T, Hishima T, Koizumi $\mathrm{F}$, Kanemasa $\mathrm{Y}$, et al. Long-term complete remission in a patient with intravascular large B-cell lymphoma with central nervous system involvement. Onco Targets Ther. 2014;7:2133-6.

This case report considers unique drug interaction and role of verapamil in favorable outcome of IVL BCL diagnosed with CNS involvement.

29. Iwamuro M, Kimura K, Kondo E, Otsuka F. Hyperintense lesion in the pons in intravascular lymphoma. Intern Med. 2015;54:2421-2.

30. Miller TP, Grogan TM, Dalton WS, Spier CM, Scheper RJ, Salmon SE. P-glycoprotein expression in malignant lymphoma and reversal of clinical drug resistance with chemotherapy plus high-dose verapamil. J Clin Oncol. 1991;9:17-24.

31. Cordon-Cardo C, O'Brien JP, Casals D, Rittman-Grauer L, Biedler JL, Melamed MR, et al. Multidrug-resistance gene (P-glycoprotein) is expressed by endothelial cells at blood-brain barrier sites. Proc Natl Acad Sci U S A. 1989;86:695-8.

32. Abbasi H, Bell SL, Stewart W, Neelakantan A, Webb S. A mystery solved. Pract Neurol. 2014;14:107-9.

33. Chaturvedi S, Pant I, Kushwaha S, Jha DK (2014) Intravascular lymphoma: an unusual cause of rapid cognitive decline and the role of brain biopsy. Case Reports 2014:bcr2014205835-bcr2014205835.
34. Panda A, Malik S. CNS intravascular lymphoma: an underappreciated cause of rapidly progressive dementia. BMJ Case Rep. 2014; doi:10.1136/bcr-2014-203772.

35. Baehring JM, Longtine J, Hochberg FH (2003) A new approach to the diagnosis and treatment of intravascular lymphoma. 237-248.

36. Kloc G, Budziak M, Więckiewicz A, Pleśniak M, Bartosik-Psujek H. Intravascular lymphoma mimicking multiple sclerosis. Neurol Neurochir Pol. 2016;50:313-7.

37. Kebir S, Kuchelmeister K, Niehusmann P, et al. Intravascular CNS lymphoma: successful therapy using high-dose methotrexate-based polychemotherapy. Exp Hematol Oncol. 2012;1:37.

38. Pels H, Schmidt-Wolf IGH, Glasmacher A, et al. Primary central nervous system lymphoma: results of a pilot and phase II study of systemic and intraventricular chemotherapy with deferred radiotherapy. J Clin Oncol. 2003;21:4489-95.

39. Prats-Martín C, Franco-Macías E, Morales-Camacho RM, Pérez O, Vargas MT, de la Cruz VF, et al. Intravascular lymphoma: look through the small vessels. Ann Hematol. 2017;96:517-9.

40. Momota H, Narita Y, Miyakita Y, Shibui S. Intravascular lymphoma of the central nervous system presenting as multiple cerebral infarctions. Nagoya J Med Sci. 2012;74:353-8.

41. Cruto C, Taipa R, Monteiro C, Moreira I, Melo-Pires M, Correia M. Multiple cerebral infarcts and intravascular central nervous system lymphoma: a rare but potentially treatable association. J Neurol Sci. 2013;325:183-5.

42. Hung L, Tsai J, Wu C, Dai Y, Chen C (2014) Brain biopsy-proven intravascular lymphomatosis presenting as rapidly recurrent strokes-two case reports. 23:11-18.

43. Kawabata H, Setoguchi T, Yone K, Souda M, Yoshida $\mathrm{H}$, Kawahara KI, et al. High mobility group box 1 is upregulated after spinal cord injury and is associated with neuronal cell apoptosis. Spine (Phila Pa 1976). 2010; doi:10.1097/BRS.0b013e3181bd14b6.

44.• Yoshida S, Kuroda H, Fukuhara N, Konno H, Watanabe $\mathrm{M}$, Akaishi T, et al. Heparin-responsive angiopathy in the central nervous system caused by intravascular large B-cell lymphoma. J Neurol Sci. 2015;352:117-9.

This manuscript discusses novel contribution of heparincombined therapy for treatment of IVL BCL.

45. Kanda M, Suzumiya J, Ohshima K, Tamura K, Kikuchi M. Intravascular large cell lymphoma: clinicopathological, immuno-histochemical and molecular genetic studies. Leuk Lymphoma. 1999;34:569-80. 